\title{
An alternative strategy for coronary artery lesions with an extra-large reference diameter using a perfusion balloon
}

\author{
Satoshi Asada, Kenichi Sakakura, Kei Yamamoto, Shinichi Momomura, Hideo Fujita \\ Division of Cardiovascular Medicine, Saitama Medical Center, Jichi Medical University, Saitama City, Japan
}

Adv Interv Cardiol 2020; 16, 2 (60): 219-220

DOI: https://doi.org/10.5114/aic.2020.96069

Although drug-eluting stents (DES) have dramatically reduced the target vessel revascularization in contemporary percutaneous coronary interventions $(\mathrm{PCl})$, revascularization to a lesion with an extra-large reference diameter is still challenging. Since the maximum size of DES is limited to $4.0 \mathrm{~mm}$ in Japan, stent malapposition would be inevitable if we used a DES for a lesion with a reference diameter of $\geq 5.5-6.0 \mathrm{~mm}$. The stent-less strategy such as the drug-coated balloon (DCB) following scoring balloon dilatation may be a good substitute for lesions with an extra-large reference diameter. However, bailout stenting is sometimes necessary even in the planned stent-less strategy [1]. Therefore, further refinements and discussions are needed for such lesions. In this case report, we show the utility of the perfusion balloon for lesions with an extra-large reference diameter.

An 80s-year-old man was referred to our hospital for $\mathrm{PCl}$. Coronary angiography showed a stenosis in the proximal segment of the right coronary artery (RCA), which had an extra-large reference diameter (Figure $1 \mathrm{~A}$ ). Intravascular ultrasound (IVUS) revealed that the proximal reference diameter, the minimum lumen diameter and distal reference diameter were $5.3 \mathrm{~mm}, 2.3 \mathrm{~mm}$, and $6.7 \mathrm{~mm}$, respectively (Figures $1 \mathrm{~B}-\mathrm{D}$ ). We performed a prolonged period of balloon dilatation to the lesion for 10 min using a $4.0 \times 20 \mathrm{~mm}$ perfusion balloon with $6 \mathrm{~atm}$ (Ryusei: Kaneka, Osaka, Japan) (Figures 1 E, F). After 10 min of dilatation, a sufficient lumen area was obtained without significant dissections (Figure $1 \mathrm{G}$ ). After an additional 10 min of observation, we finished the procedure without DES or DCB (Figure $1 \mathrm{H}$ ). After 6 months, follow-up coronary angiography revealed good patency of the lesion (Figure $1 \mathrm{l}$ ).
The perfusion balloon has been used as a bailout device in cases of vessel perforation, occlusive dissections, and thrombotic occlusions [2]. A unique characteristic of the perfusion balloon is to maintain blood flow during balloon inflation, which allowed us to perform a prolonged period of balloon dilatation for $10 \mathrm{~min}$. Recently, Horie et al. reported that prolonged balloon dilatation $(7.8 \pm 2.7 \mathrm{~min})$ was inversely associated with severe dissection in the revascularization of femoropopliteal lesions [3]. However, unlike femoropopliteal lesions, such prolonged inflation to coronary lesions is difficult to perform except by perfusion balloon. Although we obtained an optimal result, we should mention the possibility that prolonged balloon dilatation might not work. If prolonged balloon dilatation provoked severe dissections, we would have to deploy a stent for bailout, which would cause stent malapposition. Furthermore, if we applied larger stents (> $5.0 \mathrm{~mm}$ ) to avoid stent malapposition, we might have a vessel perforation, because the culprit vessel diameter was less than $5 \mathrm{~mm}$ (Figure $1 \mathrm{C}$ ). Self-expandable stents, which were not available in Japan, might be the choice. In summary, our case provides an alternative option for coronary lesions with an extra-large reference diameter using a perfusion balloon.

\section{Conflict of interest}

Dr. Sakakura has received speaking honoraria from Abbott Vascular, Boston Scientific, Medtronic Cardiovascular, Terumo, OrbusNeich, Japan Lifeline, and NIPRO. He has served as a proctor for Rotablator for Boston Scientific and has served as a consultant for Abbott Vascular and Boston Scientific. Prof. Fujita served as a consultant for Mehergen Group Holdings, Inc.

\section{Corresponding author:}

Kenichi Sakakura MD, Division of Cardiovascular Medicine, Saitama Medical Center, Jichi Medical University, 1-847 Amanuma, Omiya, 330-8503 Saitama City, Japan, phone: +81 48 647-2111, fax: +81 48648 5188, e-mail: ksakakura@jichi.ac.jp

Received: 18.03.2020, accepted: 8.04.2020. 

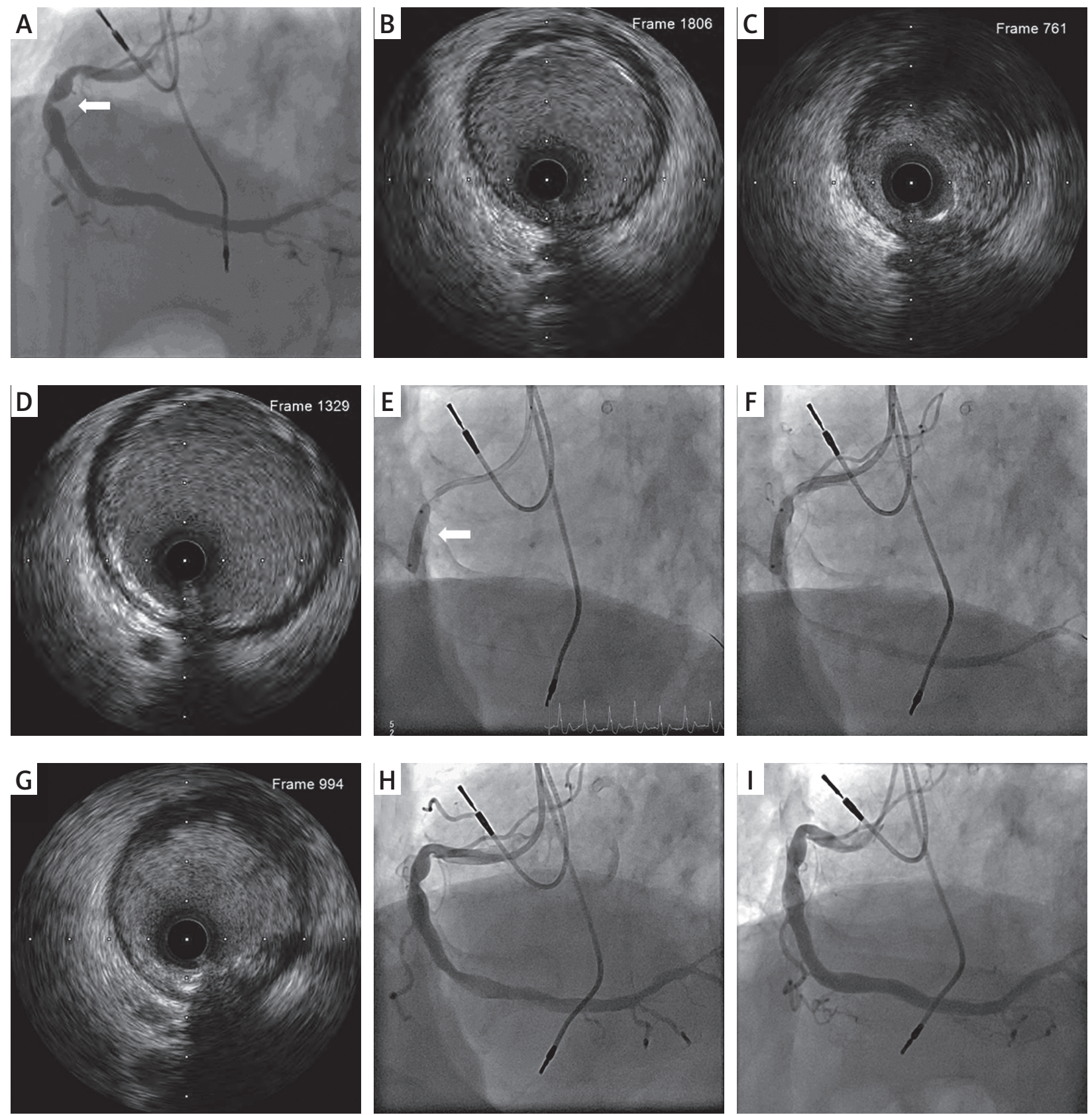

Figure 1. A - Lesion (white arrow) with extra-large references at both ends. B - Proximal reference $(5.3 \mathrm{~mm})$. C - Minimum lumen. D - Distal reference $(6.7 \mathrm{~mm})$. E - $4.0 \times 20 \mathrm{~mm}$ Perfusion balloon (white arrow). F - Distal coronary flow was maintained during perfusion balloon dilatation. Dilatation using a perfusion balloon. G - Post IVUS did not show severe dissection. H - Final angiogram. I - Six-months follow-up angiogram

\section{References}

1. Nishiyama N, Komatsu T, Kuroyanagi T, et al. Clinical value of drug-coated balloon angioplasty for de novo lesions in patients with coronary artery disease. Int J Cardiol 2016; 222: 113-8.

2. Seggewiss H, Gleichmann U, Fassbender D, et al. Therapy for acute vascular complications in percutaneous transluminal coronary angioplasty with the autoperfusion balloon catheter. Eur Heart J 1992; 13: 1649-57.

3. Horie K, Tanaka A, Taguri M, et al. Impact of prolonged inflation times during plain balloon angioplasty on angiographic dissection in femoropopliteal lesions. J Endovasc Ther 2018; 25: 683-91. 